Instituto Internacional de Investigación y Desarrollo Tecnológico Educativo INDTEC, C.A.

DOI: https://doi.org/10.29394/Scientific.issn.2542-2987.2018.3.8.17.319-333

OAI-PMH: http://www.indteca.com/ojs/index.php/Revista Scientific/oai

\title{
Visión de la Gestión en la Gerencia de la Responsabilidad Social Universitaria
}

\author{
Autor: Juan Carlos Pernía \\ Universidad Centroccidental Lisandro Alvarado, UCLA \\ jpernia@ucla.edu.ve \\ Lara, Venezuela
}

\section{Resumen}

Las ciencias administrativas en las gestiones gerenciales generan espacios para la investigación sobre la contribución de las universidades en la formación de profesionales socialmente responsables. El propósito de este ensayo es generar una reflexión acerca de la visión de la gestión en la gerencia de la Responsabilidad Social Universitaria (RSU). El proceso metódico utilizado para el acopio de la información, se apoya en la revisión documental de algunos esbozos teóricos relacionados con los constructos de la RSU, como el cuidado, manejo, utilización de los bienes y servicios en procura del beneficio del bien común de todos los ciudadanos; las cuales deben orientarse hacia nuevas alternativas gerenciales que sean más eficientes para adaptarse a la dinámica compleja del crecimiento de la sociedad. De ahí que las organizaciones de educación superior, tienen una corresponsabilidad de tomar las medidas de protección de medio ambiente, además de contribuir con el bienestar de la sociedad, como un todo mancomunado que debe responder a los intereses organizacionales, pero también del desarrollo sustentable del país. Se concluyó que la responsabilidad social universitaria debe promover una perspectiva reflexiva de los gerentes académicos para trascender la labor formativa, desde una mirada prospectiva estratégica en un pensamiento sistémico, sin barreras disciplinaria que asuma una actitud desestructurada de la complejidad, creando nuevos espacios de gestión presente y futura de la organización académica orientada a egresar profesionales con conocimientos pertinentes a las demandas de la sociedad contemporánea, personas comprometidas con mejorar la calidad de vida colectiva desde una visión planetaria.

Palabras clave: gestión; prospectiva; responsabilidad social; universidad. 


\title{
Vision of Management in the Management of University Social Responsibility
}

\begin{abstract}
The administrative and managerial sciences generate spaces for research on the contribution of universities in the training of socially responsible professionals. The purpose of this essay is to generate a reflection on the management view of the University Social Responsibility (RSU). The methodical process used to collect the information is supported by the documentary review of some theoretical sketches related to the constructs of the RSU, such as care, management, use of goods and services in pursuit of the benefit of the common good of all the citizens; which should be oriented towards new managerial alternatives that are more efficient to adapt to the complex dynamics of society's growth. Hence, higher education organizations have the responsibility to take measures to protect the environment, in addition to contributing to the welfare of society, as a whole that must respond to organizational interests but also the sustainable development of the country. It was concluded that university social responsibility should focus on promoting a critical and reflective approach by academic managers to go beyond their training jobs, without disciplinary barriers that assume an unstructured attitude of complexity, creating new spaces of present management and future of the academic organization aimed at graduating professionals with relevant knowledge to the demands of contemporary society, people committed to improving the quality of collective life from a planetary view.
\end{abstract}

Keywords: management; prospective; social responsibility; university. 


\section{Introducción}

Las sociedades actuales se encuentran en un proceso de transición, de una actividad productiva y económica basada especialmente en la industria a una basada en el conocimiento, en donde el procesamiento de la información

y la comunicación de símbolos pasan a ser la principal actividad productiva y de desarrollo económico y social a través de la formación de los profesionales capaces de generar planes estratégicos de desarrollo de la sociedad contemporánea.

Los planes estratégicos de la gerencia deben estar orientados a garantizar el suministro de recursos necesarios para la satisfacción de sus necesidades sentidas que englobe todos los aspectos de la vida de los ciudadanos. Éstos deben estar vinculados en forma y contenido, a los aspectos sociales, por supuesto debe contemplar lo económico en términos de racionalización estratégica de sustentabilidad.

La racionalidad estratégica del gerente, tiene su base táctica en las habilidades personales, con dominio técnico-administrativa-humanas para proyectar el futuro deseado. Se trata de visión de futuro capaz de formar profesionales con un perfil axiológico para asumir la responsabilidad social a través de acciones Integrales a través de vínculos complejos de relaciones, que además de ser aleatorios, tienen efectos recíprocos dentro de las organizaciones universitarias con la sociedad a la cual pertenecen desde una perspectiva planetaria de convivencia.

En este sentido, El propósito de este ensayo es generar una reflexión acerca de la visión de la gestión en la gerencia de la Responsabilidad Social Universitaria (RSU). El proceso metódico utilizado para el acopio de la información que fundamenta este estudio, se apoya en la revisión documental de algunos esbozos relacionados con los constructos teóricos de estudio, los cuales se presenta a continuación. 


\section{Análisis de los Planteamientos Teóricos}

Las tendencias, sociales, políticas y administrativas durante las últimas décadas, han creado nuevas dinámicas en las organizaciones, las cuales deben orientarse hacia nuevas alternativas gerenciales avanzadas que sean más eficientes para adaptarse a la dinámica compleja del crecimiento de la sociedad de tal modo, de ir adecuándose a los cambios científicos y técnicos que le permitan el manejo de los procesos en la búsqueda de la eficacia, de las operaciones tecno-administrativa-productivas, en términos de apoyar la gestión de los gerentes dentro de las instituciones públicas o privadas como es el caso de las universidades.

De acuerdo con los planteamientos de Drucker (2012), en la administración de la organización universitaria:

La gerencia es un cargo que ocupa el director de una empresa lo cual tiene dentro de sus múltiples funciones, además de representar a la sociedad frente a terceros y coordinar todos los recursos a través del proceso de planeamiento, organización dirección y control con el fin de lograr objetivos establecidos (pág. 97).

Por lo tanto, dentro de esta dinámica el gerente en la administración pública en muchos casos cumple diversas funciones que le exige desenvolverse como supervisor, contralor, auditor, entre otras actuaciones inherentes a su cargo. En este contexto gerencial, las organizaciones universitarias, también desarrollan acciones relacionadas con la dirección y control de los procesos tecno-administrativos. De acuerdo con Barroso (2000): la gestión implica los procesos de planificación, organización, selección y control, además de lidiar con la incertidumbre y complejidad de las interacciones tanto dentro, como fuera de la organización, relacionados con una gestión de calidad desde una perspectiva de responsabilidad social.

En palabras de Vega (2009): la responsabilidad social está relacionada con el "compromiso que la organización tiene con la sociedad en la que 
desarrolla sus actividades, ya sea como institución social, como organización económica particular o como el conjunto de individuos que la integran (pág. 21). En los últimos tiempos, ha adquirido una dimensión ética que toda organización o institución debería incorporar dentro de sus políticas y estructura organizativa, fungiendo como estrategias de gestión en su actividad diaria.

Según Benavides, Alameda y Villagra (2002): Ia Responsabilidad Social Empresarial (RSE), reconoce la importancia de "desarrollar sistemas de valores que reflejen la voluntad de asumir estas responsabilidades en lugar de traspasarla a otros (pág. 42). Por lo tanto, representa el conjunto estrategias corporativas, dirigidas a producir beneficios en las actividades tanto de la empresa, como de la sociedad destinadas a promover el desarrollo de la comunidad, y a generar en su talento humano mayor compromiso con su labor productiva corresponsable. Asimismo, en el ámbito universitario, Díaz (2009), afirma que:

La universidad como centro de desarrollo y promoción del conocimiento tiene la responsabilidad de propiciar una mejor toma de conciencia de los problemas mundiales como los perjuicios causados al medio ambiente, los riesgos tecnológicos, la crisis económica, la pobreza, el hambre, entre otros, mediante la promoción, en el seno de su formación, de un pensamiento complejo y plural, basado en la solidaridad, ética, cooperación-colaboración científica, humanística y tecnológica. La sociedad actual avanza en la medida en la que se genera, apropia y utiliza el conocimiento, creado u obtenido localmente, para atender a las necesidades de desarrollo y construir su propio futuro (pág. 12).

La universidad como principal productora de conocimiento adquiere una gran responsabilidad en donde se ve en la obligación de invertir recursos como lo señala Didriksson (2016a): con la finalidad de producir innovación y cambios radicales que respondan a las necesidades a nivel interno (investigación, nuevas formas de gestión y organización) y externo (contacto de la universidad 
con la sociedad), en función de las realidades actuales y su visión de futuro en estas organizaciones.

En consecuencia, visión de futuro en palabras de Godet (2007): representa una concepción prospectiva, la cual constituye una representación anticipada (preactiva), que configura una condición proactiva para gestionar desde la gerencia, las acciones deseables para alcanzar los fines de la organización. Con el propósito de contribuir a la formulación del concepto, en este documento planteo, que se trata de una disciplina que a partir del conjunto colectivo de pensamientos, intereses, sentimientos y sensaciones personales y colectivas, busca preparar los escenarios, a partir de una metodología, desde una imagen estructurada para la construcción de los proyectos de formación profesional como le corresponde a la universidad.

En el mismo orden de ideas, González y López (2014): sostienen el papel decisivo de la universidad en el desarrollo social, económico, político y cultural de un país; para lo cual López y Puevo (2016): señalan que se requiere la formación de profesionales con un alto nivel reflexivo, crítico y una base ética sólida. Tomando esta afirmación como punto de partida, podría destacarse un concepto estrechamente vinculado con el ámbito empresarial, más conocido y difundido en los últimos tiempos. Se habla de una dimensión ética organizacional con una visión de promover en su actividad diaria el bienestar colectivo.

En los últimos años, se ha evidenciado un interés creciente por parte de las comunidades y la sociedad; De Fátima, Baptista y Contreras (2012): explican se debe llevar a cabo a través de un proceso de reflexión acerca de la importancia e impacto de la participación ciudadana, la solidaridad y la ética en los entornos cada vez más complejos, donde prevalecen problemas económicos, sociales y ambientales que obstaculizan el desarrollo de las sociedades. Este sentimiento de responsabilidad acerca del impacto individual y colectivo de las acciones representa el inicio de la actividad y definición de 
la responsabilidad social.

En concordancia con Didriksson (2016b): las instituciones universitarias en Latinoamérica desde una proyección en el siglo XXI, están sometidas a las demandas sociales contemporáneas, las cuales requiere definir una visión y política claras, del liderazgo en la gestión de la gerencia de las funciones de docencia, investigación y extensión que abordan la responsabilidad social.

En correspondencia con lo planteado acerca de la responsabilidad social, desde los planteamientos de Sánchez (2007), hace "un llamado a las instituciones de enseñanza superior a reconstruir y reelaborar la cultura y el saber para desarrollar una sociedad más justa y humana" (pág. 12). Esta abstracción, conduce a las universidades a asumir una reflexión sobre su incursión en la responsabilidad social, no sólo por ser organizaciones, sino porque en su seno se forman los profesionales requeridos por las empresas, en estos ámbitos laborales promoverán una visión ética, contribuyendo con bien común y la justicia social.

Del mismo modo, Romero (2008): señala que la responsabilidad social universitaria "consiste en colocar a la persona en el centro de nuestra preocupación y llevarla a la enseñanza, a la investigación y a las decisiones que se tomen como parte de la Universidad y más allá de ella" (pág. 5). Por eso, su enfoque es integrador y complejo, llegando a enfatizar y estimular la relación con el entorno, basado en la pertinencia y compromiso social, con una reconstrucción del currículo en las necesidades emergentes junto a la integración e internacionalización de la educación.

En palabras de Vallaeys (2014a): a nivel latinoamericano se ha dado pasos agigantados, debido a su postura más integral, compleja y amplia en comparación con la práctica implementada en América del Norte y Europa. En estas zonas, se han abocado a abordar los aspectos relacionados al medio ambiente (campus sostenible), con una escasa atención a los procesos formativos o de vinculación y ninguna atención a los procesos cognitivos y 
epistemológicos.

En esta orientación, la necesidad de innovar en la formación universitaria, a través de las funciones de docencia, investigación y extensión, promueve una proyección social, así como la ampliación de la ciencia, la tecnología y la cultura nacional; defendiendo la autonomía desde una novedosa tendencia globalización de la educación superior.

La responsabilidad social universitaria se ha colocado en la actualidad como un movimiento mundial, implantado en el sistema de gestión de las casas de estudio, como una necesidad ante el desarrollo vertiginoso de la sociedad en todos los aspectos éticos, con algunos referentes axiológicos de la formación profesional Vallaeys (2014b): señala la importancia de una política educativa a través de la gestión responsable, capaz de asumir la RSU, a través de un diálogo participativo con el entorno social, para mejorar su calidad y promover un desarrollo humano sostenible.

En esta orientación Villegas (2017): señala que la responsabilidad social implica el desarrollo de "conductas y comportamientos apegados a las normativas, leyes que regulan su funcionamiento, donde puedan funcionar $\sin$ causas efectos adversos a la sociedad" (pág. 294). De ahí, la importancia de centrar el discurso académico para desplegar una formación ética de todas las personas que hacen vida en las universidades de forma tal que de acuerdo con Izarra (2016): el impacto en la gestión, en la actividad docente, en la generación de conocimiento redimensiona las actividades asociadas a la gerencia de la RSU, a través de programas de atención a las comunidades inmediatas de cada institución universitaria.

De acuerdo con Senge (2005a): se debe identificar la sensibilidad del hacer gerencial, en la búsqueda de "interconexiones sutiles que confieren a los sistemas vivientes un carácter singular" (pág. 91). Este escenario complejo debe abordase desde un pensamiento sistémico de la gerencia y por consiguiente de la gestión prospectiva de la RSU, para sostener la capacidad 
de impulsar los cambios necesarios que integran las diversas funciones, en virtud de mejorar la calidad de la formación profesional.

De esta manera, las iniciativas de Responsabilidad Social Universitaria (RSU), reconoce en la gestión de la gerencia de los proyectos de extensión, el carácter ético de la comunidad académica, debido a su incidencia en la vida cotidiana institucional y la imagen que estas casas de estudios tiene en la conformación del perfil profesional, más allá del desarrollo de los contenidos formales de experiencias dentro y fuera del aula desde una corresponsabilidad social que deben ser proyectada al futuro deseado.

Forero y Perilla (2004, pág. 14): explican que una visión prospectiva del entorno gerencial representa la habilidad para manejar la información del entorno a favor de su profesión, las organizaciones para las que labora y los individuos en general; por ello, es indispensable ver la posibilidad de armar el rompecabezas organizacional universitario, desde una nueva óptica que permita visualizar la realidad presente con una proyección de futuro, vista como una totalidad.

De allí que Senge (2005b): sostiene que "el pensamiento sistémico es una disciplina para ver modalidades" (pág. 91). Por consiguiente, sirve de marco de referencia para ver interrelaciones internas y externas, en vez de simplificar las dinámicas propias del liderazgo universitario, despertando la capacidad de los gerentes de estas instituciones para asumir una responsabilidad social.

Por ello, la responsabilidad social en la gestión de la gerencia universitaria, debe utilizar las herramientas y técnicas específicas para provocar un cambio en las acciones organizacional, así como en las que implican la tarea académica en la formación de los futuros profesionales, lo cual se traduce en la decodificación de posibles patrones de cambio en el comportamiento ético corresponsable en la organización.

Desde una perspectiva de responsabilidad social universitaria, debe 
estar destinada a desplegar una gestión académica dirigida a alcanzar los fines de la gerencia de la educación superior para responder a las demandas de la sociedad una visión de futuro coherente con la convivencia planetaria de los ciudadanos.

\section{Hallazgos de la Revisión Teórica}

El análisis de los constructos teóricos, representa la base de los resultados en este ensayo a partir de una síntesis de los elementos más significativos que contribuyen a un esquema relacional de la gerencia prospectiva de la Responsabilidad Social Universitaria (RSU), como se muestran en el gráfico 1.

Gráfico 1. Esquema relacional de la gerencia prospectiva de la RSU.

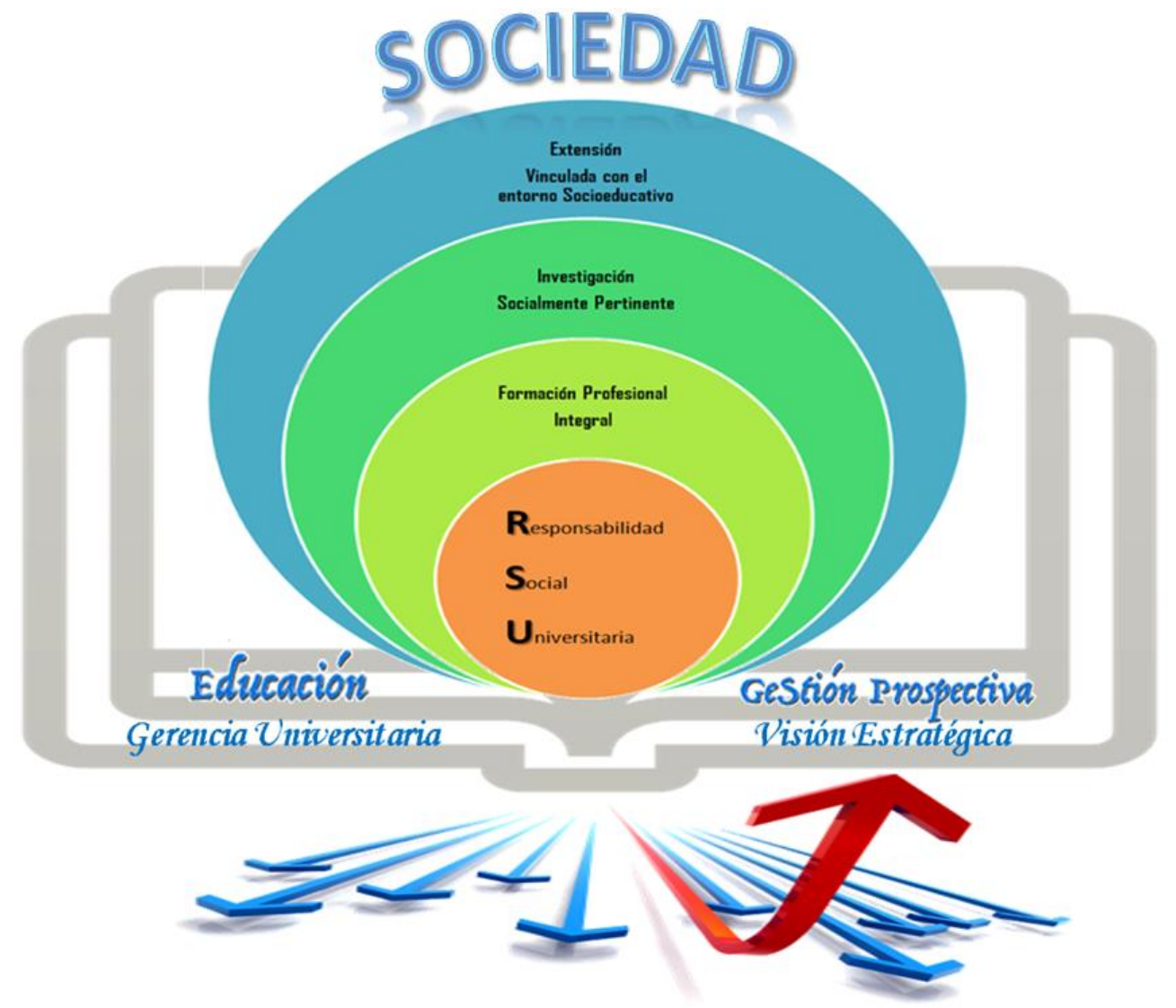

Fuente: El Autor (2017). 
El gráfico 1, muestra el conjunto esquema relacional de la gestión prospectiva en la gerencia de la Responsabilidad Social Universitaria (RSU), en lo que concierne la docencia se debe enfatizar en la capacitación de los docentes en el enfoque de la RSU, para promover en las especialidades el aprendizaje basado en proyectos con apertura a la comunidad social como fuente de enseñanza significativa y práctica aplicada a la solución de problemas reales. Asimismo, la investigación, debe promover convenios interdisciplinarios desde sus especialidades respectivas; y la extensión estaría consustanciada una sinergia de saberes articulados con las interacciones socioeducativas.

En esta connotación sinérgica, la universidad asume socialmente la responsabilidad de promover valores formativos con una visión académica organizacional ética, desde la cual, se impregna la gestión de sus funciones de docencia, investigación y extensión, en conformidad con las políticas gerenciales universitarias, las iniciativas de RSU responden axiológicamente al modelo ético que forma parte del currículum profesional, el cual está caracterizado por la acción académica corresponsable, más allá de los contenidos programáticos, sin olvidarse de la importancia de proyectarlas al futuro deseado, en términos de responder a las demandas de la sociedad globalizada.

En correspondencia con los planteamientos anteriores, la visión pedagógica de la RSU, debe estar dirigida a promover prácticas éticas desde una gerencia prospectiva, debe plantear las funciones de (a) docencia, desde una formación integral y de calidad; (b) la investigación, con abordaje pertinente a las demandas de la sociedad; y (c) una extensión, vinculada con los entornos sociales, ambientales y planetarios. En fin, se trata de integrar la labor académica orientada a egresar profesionales con conocimientos pertinentes a las demandas de la sociedad contemporánea a través de una gestión estratégica capaz de asumir una corresponsabilidad compartida. 


\section{Conclusiones}

Una vez culminada la exploración de los constructos teóricos, se llegó a las siguientes conclusiones: Primero, las competencias tecnológicas del docente para la innovación de la praxis educativa, requiere además de la vocación de servicio, estudiar los nuevos paradigmas didácticos para desplegar su capacidad reflexiva y asumir el compromiso de adquirir las habilidades tecnológicas para asumir los requerimientos de una sociedad global.

Segundo, la responsabilidad social representa un estado de conciencia, que puede darse de manera individual, asumido por cada uno de los elementos que conforman a la sociedad. Desde esta perspectiva, la ética, no aparece como freno al interés de la organización sino al contrario, como empuje para su provecho y modalidad de funcionamiento de una mayor articulación entre las funciones de docencia, investigación y extensión.

Tercero, uno de los retos que afrontan los gerentes de las organizaciones empresariales, es precisamente asumir una gestión estratégica prospectiva que oriente sus gestiones con la incorporación de otras alternativas que requieren roles cambiantes para lo que se requiere elevar los niveles de madurez organizacional.

Finalmente, la responsabilidad social universitaria debe promover una perspectiva reflexiva de los gerentes académicos para trascender la labor formativa, desde una mirada prospectiva estratégica en un pensamiento sistémico, sin barreras disciplinarias que asuma una actitud desestructurada de la complejidad, creando nuevos espacios de gestión presente y futura de la organización académica orientada a egresar profesionales con conocimientos pertinentes a las demandas de la sociedad contemporánea, personas comprometidas con mejorar la calidad de vida colectiva desde una visión planetaria. 


\section{Referencias}

Barroso, M. (2000). Meditaciones Gerenciales. Venezuela: Galoe.

Benavides J., Alameda D., \& Villagra N. (2002). La Comunicación y la Cultura en la Sociedad del Conocimiento. Madrid: UCM.

De Fátima M., Baptista, M., \& Contreras, H. (2012). La Innovación Social en el Contexto de la Responsabilidad Social Empresarial. Forum Empresarial, 17(1), 31-63. Recuperado de:

http://www.redalyc.org/articulo.oa?id=63124039002

Díaz, E. (2009). Necesidad de un Modelo de Desarrollo Económico Sustentable. Revista Forestal Latinoamericana, Vol. 019 (1), №35. Mérida, Venezuela: SABER-ULA, Universidad de los Andes, págs. 6578. Recuperado de: http://www.saber.ula.ve/handle/123456789/24116

Didriksson, A. (2016a,b). La Universidad desde su Futuro. Brasil: Proposições.

Drucker, P. (2012). Principios de la Organización Administrativa. México: Prentice Hall.

Forero E., \& Perilla L. (2004). Visión Retrospectiva, Actual y Prospectiva de la Psicología Organizacional. Colombia: Universidad Católica De Colombia.

Godet, M. (2007). Prospectiva Estratégica: problemas y métodos. Por Michael Godet en colaboración con Philippe Durance y la participación de Prospektiker. Cuaderno no 20. Segunda edición. Cuadernos de LIPSOR. Instituto Europeo de Prospectiva y Estrategia. París: PROSPEKTIKER. Recuperado de:

http://www.laprospective.fr/dyn/francais/memoire/Cajadeherramientas2 $\underline{007 . p d f}$

González, K., \& López, J. (2014). Responsabilidad Social Universitaria. Apuntes para un Modelo de RSU. Revista de Comunicación Centro Gumilla. Revista de Comunicación 13, págs. 84-117. Recuperado de: 
https://dialnet.unirioja.es/descarga/articulo/4875638.pdf

Izarra, D. (2016). Experiencias y Perspectivas en Ética Profesional y Responsabilidad Social Universitaria en Iberoamérica. Caracas: Universidad Pedagógica Experimental Libertador Centro de Investigación Educativa Georgina Calderón.

López, R., \& Puevo, M. (2016). La Universidad y las Profesiones. Murcia, España: Educatio Siglo XXI.

Romero, C. (2008). Sobre la Responsabilidad Social en la PUCP. Lima, Perú: Editorial Pontificia Universidad Católica del Perú.

Sánchez, C. (2007). La Responsabilidad Social Universitaria (RSU) en el Contexto del Cambio de la Educación Superior. Brasil: Virtual Educa. Senge, P. (2005a,b). La Quinta Disciplina. El Arte y la Práctica de la Organización Abierta al Aprendizaje. Buenos Aires: Granica.

Vallaeys, F. (2014a,b). La Responsabilidad Social Universitaria: Un Nuevo Modelo Universitario contra la Mercantilización. México: McGrawHill.

Vega, J. (2009). La Responsabilidad Social y los Principios del Desarrollo Sostenible como Fundamentos Teóricos de la Información Social de la Empresa. Madrid: ESIC Editorial

Villegas, Y. (2017). Responsabilidad Social Empresarial en el Contexto de las Relaciones Laborales en las PYMES. Revista Scientific, 2(6), 286302. Recuperado de:

https://doi.org/10.29394/scientific.issn.2542-2987.2017.2.6.15.286-302 


\section{Juan Carlos Pernía}

e-mail: jpernia@ucla.edu.ve

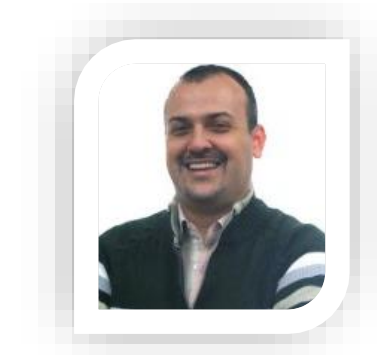

Nacido en San Cristóbal, estado Táchira, Venezuela. Abogado Universidad Católica del Táchira, Magister Scientiarum Contaduría Mención Auditoria en la Universidad Centroccidental "Lisandro Alvarado" (UCLA). Catedrático Docente Universitario por Concurso de Oposición Categoría Profesor Asociado. Locutor Certificado Universidad Central de Venezuela. Doctorando en Gerencia Avanzada de la Universidad Fermín Toro (UFT). Investigador.

El contenido de este manuscrito se difunde bajo una Licencia de Creative Commons ReconocimientoNoComercial-Compartirlgual 4.0 Internacional 